\title{
MONITORING OF COLLAPSED BUILT-UP AREAS WITH HIGH RESOLUTION SAR IMAGES
}

\author{
Raffaella Guida ${ }^{1}$, Antonio Iodice ${ }^{2}$, Daniele Riccio ${ }^{2}$ \\ ${ }^{1}$ Surrey Space Centre, University of Surrey, Guildford, UK \\ ${ }^{2}$ DIBET, Università di Napoli Federico II, Napoli, Italy
}

\begin{abstract}
A new concept for change detection algorithm for urban areas affected by earthquake is here presented. It is characterized as applicable to just one post-event amplitude Synthetic Aperture Radars (SAR) image and employs the inversion of sound scattering models already introduced in literature by the same authors. Aim of the algorithm is to try obtaining fast mapping of damaged areas and provide a first, even rough, evaluation of damage reported.

In particular in this paper the overall block diagram chain and the algorithm rationale behind that framework are introduced and discussed in details. Some preliminary results are presented and the performance analyzed. New possible applications based on similar rationale are also commented.
\end{abstract}

Index Terms - Synthetic Aperture Radar (SAR), urban areas, disaster monitoring, earthquakes.

\section{INTRODUCTION}

When natural hazards happen in built-up areas, there is an urgent need to have a map of affected areas in quick times to allow a responsive intervention. More important is the possibility to fast pinpointing damaged parts as roads (to advise about conditions and alternative road system) and buildings (to promptly save trapped human beings or warn about vital social services no-more available like hospitals). In case of earthquakes an adequate answer to most of the above needs may come from Synthetic Aperture Radar (SAR) imagery and techniques. Often, SAR interferometry is applied to get information about where land movements occurred [1] and measures of displacements in the wavelength order can be given. This kind of information, even if relevant for many aims, it is not useful for a responsive support to civil protection as not able to say how much specific areas have been hit in terms of damaged or collapsed buildings which indeed is probably the most urgent information.

In this paper we deal with the issue of identifying damaged or collapsed buildings in areas hit by earthquakes with one post-event amplitude SAR image. When a preevent SAR amplitude image of the same area is also available, an algorithm able to detect the target areas is applied by exploiting the theory based on the deterministic feature extraction approach [2] properly modified and reformulated in [3] to address a change detection problem.

The theory in [3] is then the basis of the block diagram here presented which has been conceived with the idea of developing new SAR products for supporting prompt intervention in case of natural disasters. A first application of the above concept to real SAR images has already been presented in [4] and here new results from a larger area of the same site are presented.

\section{MODELS AND THEORETICAL FRAMEWORK}

It is widely known that buildings signature in SAR images is very complex and it started appearing even more intricate with the advent of high resolution SAR images. But, whatever is the complexity of a building backscattering, a quite always present and distinctive mark can certainly be detected on SAR images of urban areas and it is given by the double bounce contribution. Closely related to the radar acquisition mode and the geometric and electromagnetic properties of the dihedral causing it, the double reflection contribution has been used by the authors for retrieving information about urban scenes [5-7], the main advantage being the use of only one SAR image.

On the other side, the analytical models there inverted, which ensured a very good description of the particular geometry considered, require the a-priori knowledge of many scene parameters which might not be given [2]. In this paper the authors try to identify those scenarios and applications which most benefit from the use of a single SAR image and that, at the same time, allow a good inversion of models adopted even when the knowledge of the scene under study is poor. 
The earthquake event in urban areas is certainly a scenario needing prompt intervention. In particular, in case of building collapse, a change in the building dihedral configuration should be reflected in a change in the corresponding double bounce signature. Here we deal with four main cases, representative of somehow canonical cases, even if we are aware they do not cover all situations:

(a) Building not reporting any damage;

(b) Building reporting partial damage;

(c) Building definitely collapsed along the side visible from the radar;

(d) Building collapsed along the side which is not in the radar line of sight.

In cases (b) and (c) a change in the double bounce signature is obviously expected in the post-event SAR image (both brightness and extension may be affected). In cases (a) and (d) instead, no change is expected (apart those due to calibration or slightly changes in the acquisition process), even when, as in case (d), a damage indeed occurred. This is the case of false negatives: since the use of just one postevent SAR image is proposed in this study some false negatives (i.e. when collapsed buildings are not detected) are likely to be present and this happens when the side affected is not the one facing the radar. In this case, since other changes happen in the building appearance in the SAR image (for example in the roof backscattering) they can be used as supporting information to limit false negatives.

So far we only dealt with geometrical changes of the buildings in the SAR image which means a geometrical change in its dihedral configuration. Nothing has been said yet about changes in electromagnetic properties or ground roughness. We here suppose that, should these change happen, they are not relevant compared with changes in the geometry. If this can be generally considered true for the dielectric properties of the surface, less true it is for the roughness with the soil in front of a collapsed building mainly occupied with the debris coming from the building. In this initial study we then mainly consider imploded buildings with the aim of successively understand how to deal with more complicated cases. This assumption allows overcoming the problem of unavailable a-priori knowledge [2] since, exploiting the theory in [3], the ratio of the preand post-event SAR images will be sufficient to know the most heavily affected areas.

Based on these considerations the concept of a new change detection algorithm has been developed and is presented in the next section.

\section{CHANGE DETECTION ALGORITHM}

The pre- and post-event amplitude SAR image of the scene under study are inputs for the blocks diagram represented in Fig.1.

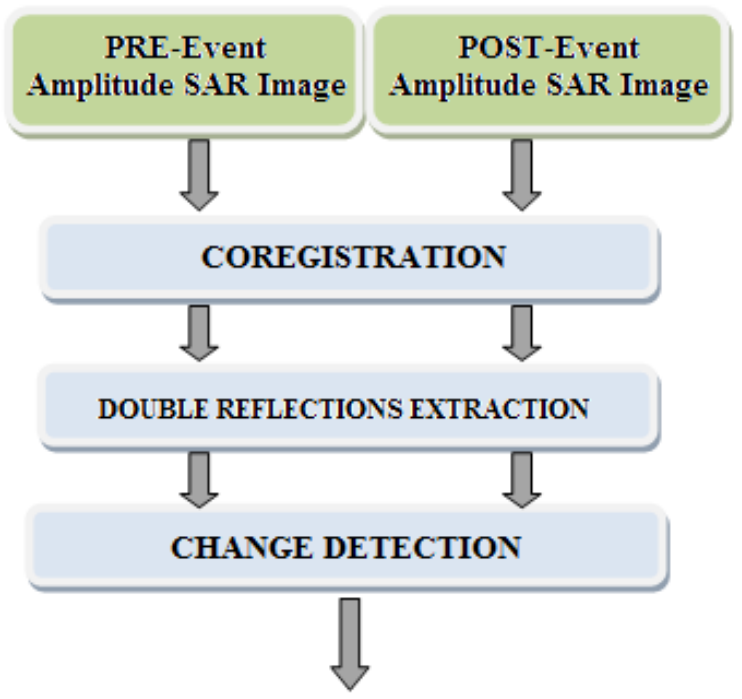

Map of damaged buildings

Figure 1 - Blocks Diagram of the change detection algorithm

The two images need to be obtained in similar conditions, with quite the same radar and orbit parameters. In the first block they are co-registered and then sent to the block performing the double reflections extraction which is detailed in Fig. 2 for a better understanding. Here, double reflection contributions are localized on the pre-event image, currently with the use of edge detection filters but further work is in progress to optimize this step. Indeed, the discrimination of double reflections is not a trivial task as detecting bright lines is not enough. Many other contributions might appear very bright and exactly in the surroundings of where the double reflection is expected (for example the strong backscattering from a sloped roof) and contextual information should be used to distinguish bright lines caused by dihedrals from those caused by other geometries or configurations.

When the double reflections are localized a mask is created and multiplied by the ratio of the pre-event image over the post-event image in order to exactly localize the double reflection ratios also in this image. The use of a mask is crucial as in the ratio image all kinds of noise are emphasized, speckle, side lobes and processing effects, [3]. The double reflections extraction block serves two main aims: the first is the extraction of the calibration constant which is got inputting in the block a built-up area not affected according to the theory and formulas presented in [3]; the second is creating a map of contributions of interest where the following block will look for possible changes occurred. 


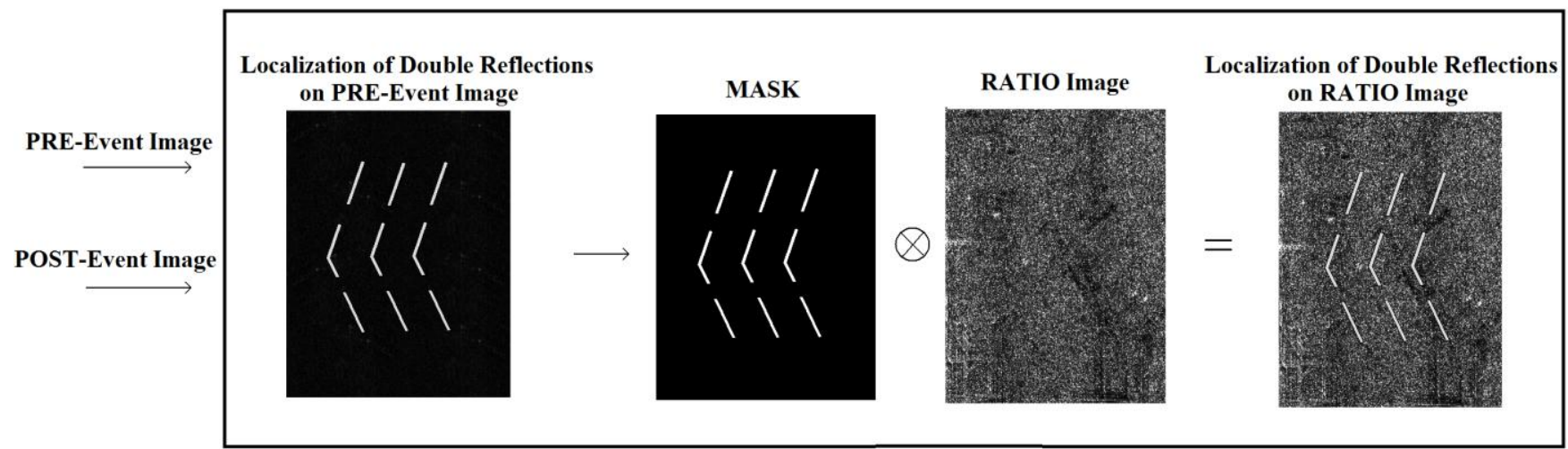

Figure 2 - Double Reflections Extraction Block

Indeed the output of this block is input for the mere change detection block, see Fig.1. Now the radiometric analysis of the contributions spotted in the previous block is performed. The average mean of each contribution is considered. Any appreciable change from the general calibration constant will be source of alarm and will be related to a certain level of damage. Finally, a map will be produced presenting exact places where the buildings are damaged and the percentage of damage.

In order to better control the noise arising in the ratio image we first applied this chain to simulated images [3] getting excellent results. Then we started considering actual cases as SAR images on L'Aquila earthquake occurred in 2009 and some still good results on a small area in L'Aquila have been presented in [4]. Next section presents results from a larger area of the same site.

\section{RESULTS}

The Italian Space Agency (ASI) kindly provided us with the pre- and post-event high resolution SAR images acquired by the Italian mission COSMO-SkyMed on the area of L'Aquila interested by a 5,8 Richter magnitude earthquake on $6^{\text {th }}$ April 2009. The available images have been acquired in the spotlight mode and present 1 meter resolution.

The chain in Figs.1-2 has been applied to the centre of the city where some buildings were severely damaged or totally collapsed.

In Fig.3a the output of the change detection block is shown. A post-event optical image, Fig.3b, acquired soon after the earthquake, is also provided to clearly indicate some of the most damaged areas.

For all three sites a heavy damage has been reported and precisely:

-site 1, damage $99.0 \%$

-site 2, damage $98.8 \%$

-site 3, damage $98.8 \%$.

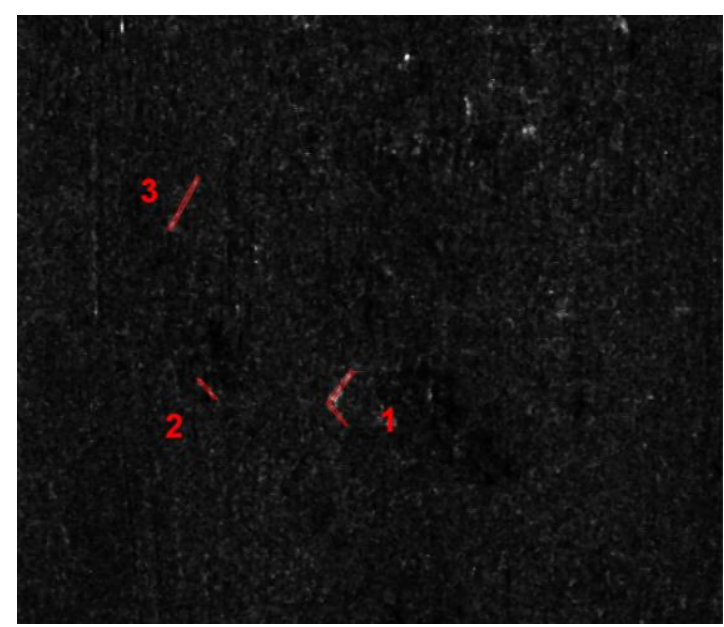

(a)

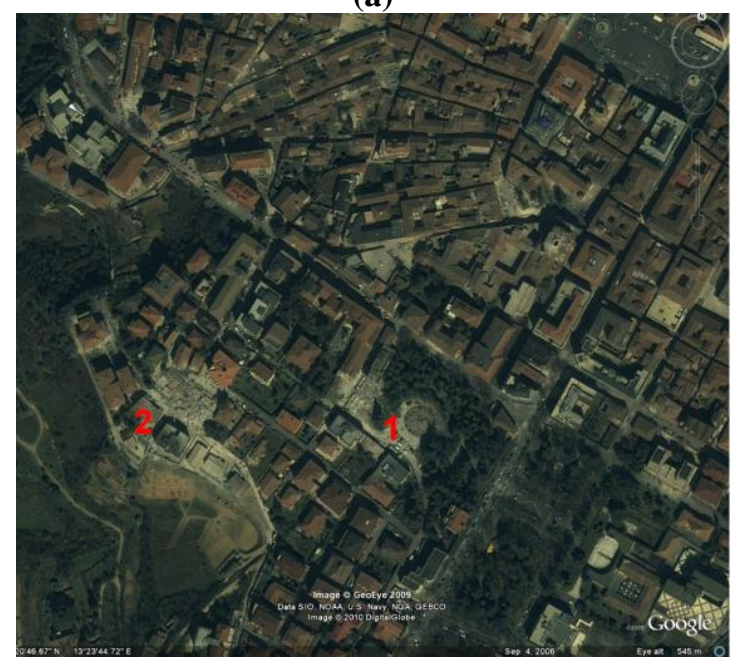

(b)

Figure 3 - Ratio of pre-event over post-event image SAR images (a), processed from products COSMO-SkyMed - OASI Agenzia Spaziale Italiana - (2009); post-event optical image of the site. 
Both site 1 and 2 have been clearly identified in the optical image where evidence is given of areas with collapsed buildings. Also the percentages of damage seem consistent with the damage shown in the picture.

Site 3 is still under investigation as the optical counterpart has not been precisely localized. Unfortunately, ground truth is not available for a double check.

\section{CONCLUSIONS}

In this paper we present the different blocks of the change detection algorithm we developed for urban areas affected by an earthquake.

A drawback generally presented by the proposed approach based on models is the high a-priori knowledge required in terms of scene parameters. But this time we proposed a solution identifying an application where simplifications in the models are possible. This resulted in a simple and elegant block diagram here presented. The idea is based on the exploitation of only one post-event SAR image or, in other words, a single couple of pre- and postevent SAR images. Being the rationale of change detection based on the analysis of change in the brightness of double reflection contributions caused by dihedrals presented by buildings, only the amplitude information in the SAR image is used.

Working with the ratio of the two input images presents the advantage of a very simple operation which preserves the integrity of radiometry which is crucial for the approach. For the same reason, use of single look complex images is suggested.

A quantitative analysis of changes in the image radiometry allows not only the detection of the damage but also its assessment as has been shown on an actual case employing high resolution COSMO SkyMed. The results shown are really promising but further study is required to localize some contributions and to assess the robustness of the approach.

Moreover new applications can be derived from the same rationale with slight adjustments. Monitoring unauthorized buildings is possible in principle using exactly the same blocks diagram but obviously, this time, an increase in the corresponding double reflection brightness is expected. in the post-event SAR image due to an increase of the dihedral surface.

The authors are also investigating another interesting application which is the monitoring of floods in urban areas and the assessment of the flood level. But in this case not all previous simplifications are possible as pre- and post-event SAR images appear very different because of water presence.

In conclusion this work shows again the flexibility of the feature extraction approach introduced by the authors in [2] and here adapted to work simply and fast on a change detection problem. The authors are currently working on the optimization of each block and the parallelization of the code for an even faster implementation.

\section{ACKNOWLEDGEMENTS}

The authors thank the Italian Space Agency (ASI) for providing the COSMO SkyMed data used in this research.

\section{REFERENCES}

[1] S.Salvi, S.Vignoli, M.Serra, V.Bosi, "Use of Cosmo-Skymed data for seismic risk management in the framework of the ASISIGRIS project", Proceedings of the International Geoscience and Remote Sensing Symposium, pp.II-921-II-924, Cape Town, (Africa), 2009.

[2]R.Guida, A.Iodice, D.Riccio, "Height Retrieval of Isolated Buildings from Single High Resolution SAR Images", IEEE Transactions on Geoscience and Remote Sensing, vol.48, n.7, pp.2967-2979, 2010.

[3]R.Guida, A.Iodice, D.Riccio, "Fast Change Detection Algorithm for Single Post-Earthquake SAR Images of Urban Areas", XVIII Riunione Nazionale di Elettromagnetismo, Benevento, accepted.

[4]R.Guida, A.Iodice, D.Riccio, "An application of the deterministic feature extraction approach to COSMO-SKyMed data, European Conference on Synthetic Aperture Radar, pp.10141017, Aachen, (Germany), June 2010.

[5] R.Guida, G.Franceschetti, A.Iodice, D.Riccio, G.Ruello, U.Stilla, "Building Feature Extraction via a Deterministic Approach: Application to Real High Resolution SAR Images", Proceedings of the International Geoscience and Remote Sensing Symposium, pp.2681-2684, Barcelona (Spain), 2007.

[6] R.Guida, A.Iodice, D.Riccio, "Assessment of TerraSAR-X Products with a New Feature Extraction Application: Monitoring of Cylindrical Tanks", IEEE Transactions on Geoscience and Remote Sensing, vol.48, n.2, pp.930-938, 2010.

[7] R.Guida, A.Iodice, D.Riccio, U.Stilla, "Model-Based Interpretation Of High Resolution SAR Images Of Buildings", IEEE Journal of Selected Topics in Applied Earth Observations and Remote Sensing, vol.1, no.2, pp.107-119, 2008. 